\title{
Diagnóstico de Falha de Curto-Circuito em Máquinas Assíncronas Utilizando Análise da Componente Principal da Corrente Transitória
}

\author{
Isabela Oliveira Zaparoli* Lane Maria Rabelo Baccarini ${ }^{* *}$ \\ Paulo Cezar Monteiro Lamim Filho*** \\ Fabiano Bianchini Batista ${ }^{* * * *}$ \\ * Universidade Federal de São João Del Rei, Brasil, \\ (oliveirazaparoli@gmail.com) \\ ** Universidade Federal de São João Del Rei, Brasil \\ (rabelo@ufsj.edu.br) \\ *** Universidade Federal de São João Del Rei, Brasil \\ (lamim@ufsj.edu.br) \\ **** Universidade Federal de São João Del Rei, Brasil \\ (fabianchini@ufsj.edu.br)
}

\begin{abstract}
The induction motor is a rugged machine and has numerous advantages as it can be exposed to a wide variety of environments and cycles of operations. However, several faults may occur during engine operation. This work proposes a methodology to diagnose the presence of initial inter-turn short-circuit of the same phase, using the motor start current signals. This analysis differs from the vast majority of the methods used, since the machine does not have to operate under nominal load conditions. The main goal is to propose a more sensitive and effective method to diagnose stator winding faults before the motor is damaged. The proposed methodology consists in using the extraction of residues from a method of signal smoothing and the Principal Component Analysis to be used to extract the behavior characteristics of the motors due to the presence of faiults.

Resumo: O motor de indução é uma máquina robusta e apresenta inúmeras vantagens, uma vez que pode ser exposto a uma ampla variedade de ambientes e ciclos de operações. Entretanto, diversas falhas podem ocorrer durante a operação do motor. Este trabalho propõe uma metodologia para diagnosticar a presença de curto-circuito inicial entre espiras de uma mesma fase utilizando os sinais da corrente de partida do motor. Esta análise se difere da grande maioria dos métodos utilizados, pois a máquina não precisa operar nas condições nominais de carga. O objetivo principal é propor um método mais sensível e eficaz para diagnosticar falhas nos enrolamentos do estator, antes que o motor seja danificado. A metodologia proposta consiste em utilizar a extração de resíduos a partir de um método de suavização do sinal, e a Análise de Componentes Principais utilizada para extrair as características de assinatura dos motores devido a presença de falhas.
\end{abstract}

Keywords: FStarting current; Inter-turn Short-circuit; Fault detection, Principal Component Analysis; Robust Extreme Envelope Average.

Palavras-chaves: Corrente de partida; Curto-circuito Entre Espiras; Detecção de falhas; Análise de Componentes Principais; Média Robusta dos Envelopes Extremos.

\section{INTRODUÇÃO}

Os motores de indução trifásicos estão amplamente presentes no nosso dia a dia. Entretanto, devido a fatores ambientais, ciclo de trabalho e/ou problemas de fabricação, falhas podem ocorrer e, se não forem detectadas em seu estágio inicial podem gerar como consequência danos irreversíveis ao motor e perdas no processo industrial. Segundo Ferreira and de Almeida (2016) e Cusido et al. (2008), os motores de indução são responsáveis por cerca de $90 \%$ dos equipamentos instalados na indústria e seu consumo energético chega a $60 \%$ do total instalado.

A grande utilização destes motores nas indústrias traz a necessidade de uma alta confiabilidade do processo. Por isso é importante conhecer as possíveis falhas que podem acontecer nessas máquinas para minimizar as interrupções não programadas Araújo et al. (2010).

Segundo Bonnett and Yung (2008), os percentuais de falhas em motores de indução trifásicos são distribuídos da seguinte forma: $69 \%$ nos rolamentos, $21 \%$ nos enrolamento 
do estator, $7 \%$ nas barra do rotor e 3\% nos eixo. Essas falhas alteram a modulação das amplitudes das correntes, da vibração, do fluxo magnético e de outras grandezas. Tais mudanças podem levar a degradação e até mesmo a quebra dos motores, consequentemente causando a interrupção do processo de produção, desperdício de material, manutenções corretivas não programadas ou troca dos mesmos em casos mais graves.

A falha de curto-circuito é uma falha severa, pois sua evolução é muito rápida. Assim, a detecção de curto-circuito inicial entre espiras é importante, pois pode evoluir para demais falhas nos enrolamentos, tais como: curto-circuito entre bobinas e curto-circuito entre fase e terra, que podem resultar em danos irreparáveis ao motor Boqiang et al. (2003). Porém, o diagnóstico desta falha não é simples, pois o curto-circuito deve ser detectado no estágio inicial e, nesse caso, o impacto nas características do motor é muito pequeno.

Atualmente, existem diversos métodos que podem ser utilizados para a detecção de curto-circuito nas espiras do estator, porém algumas técnicas propostas são extremamente invasivas e, em casos mais específicos, é necessário até que a máquina seja desligada. Por essa razão, novas metodologias para detecção prévia de falhas são necessárias de forma a não afetar a linha de produção e não colocar o operador em contato direto com o equipamento. Neste contexto, a análise da corrente do motor (Motor Current Signature Anaysis - MCSA), devido a sua característica de ser não invasiva, tem recebido grande atenção dos pesquisadores Watson and Paterson (1998).

As metodologias para análise dos sinais de corrente mais comuns utilizam o espectro das componentes do estator trabalhando em regime permanente, mas a precisão dessas técnicas dependem do percentual de carga da máquina e da relação sinal-ruído dos espectros analisados Douglas et al. (2005). Em situações em que a carga acoplada ao eixo do motor é baixa, o escorregamento diminui e a corrente que flui no estator também é menor, dificultando a detecção da falha. Por esse motivo, a análise da corrente de partida do motor pode ser utilizada como um meio complementar/alternativo para o diagnóstico de falha. Durante a partida, as máquinas assíncronas operam em condições mais críticas (correntes e estresses térmicos), algo que pode ajudar a amplificar as "assinaturas" de falhas iniciais Georgoulas et al. (2013). A principal desvantagem de usar o sinal transiente é englobar toda a gama de valores de escorregamento dentro do sinal transitório da máquina, produzindo componentes de frequência de natureza não estacionária Georgoulas et al. (2013).

Este trabalho propõe uma nova metodologia baseada na extração do resíduo através da técnica de suavização chamada média robusta dos envelopes extremos (Robust Extreme Envelope Average - REEA) e na análise de componentes principais (Principal Component Analysis - PCA), para realizar o diagnóstico de falhas de curto circuito entre espiras do estator utilizando a corrente de partida da maquina de indução trifásica. A análise da metodologia será realizada no domínio do tempo e não da frequência ou tempo frequência como em vários outros métodos.

\section{MODELO CURTO-CIRCUITO ENTRE ESPIRAS}

O modelo dinâmico assimétrico proposto por Baccarini et al. (2010), permite simular entre outras falhas o curtocircuito entre espiras do estator. A Figura 1 representa o enrolamento de uma máquina de indução com curto entre espiras.

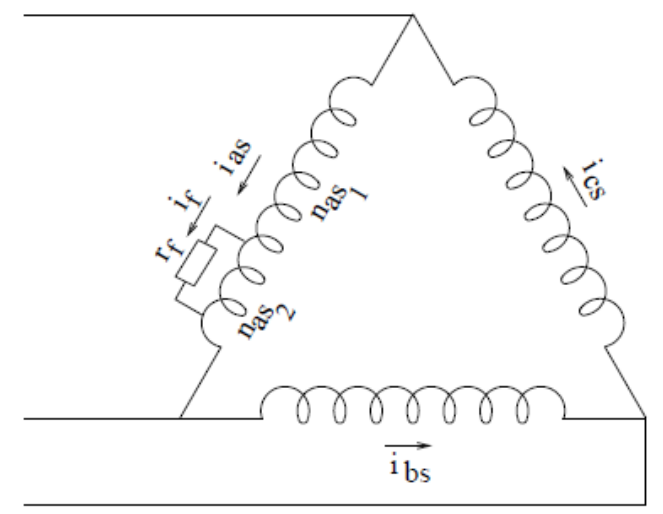

Figura 1. Representação dos enrolamentos trifásicos da máquina de indução com curto-circuito entre espiras. Retirado de Baccarini et al. (2010)

Onde $i_{f}$ é a corrente de falha; $r_{f}$ a resistência de falha; $n_{a s 1}$ o número de espiras que não estão em curto e $n_{a s 2} \mathrm{o}$ número de espiras que estão em curto; $i_{a s}, i_{b s}$ e $i_{c s}$ são as três correntes de fase do motor.

Aplicando a transformada de Park nas tensões $V a, V b$ e $V c$ do estator e considerando um percentual de espiras em curto são obtidas as Equações 1, 2 e 3, que representam as equações de estator em coordenadas $d q$. As equações do circuito do rotor são iguais às do rotor do modelo simétrico tradicional.

$$
\begin{aligned}
v_{q s}+ & \frac{2}{3} \mu r_{s} i_{f} \cos \theta=r_{s} i_{q s}+\frac{d \lambda_{q s}}{d t}+\omega \lambda_{d s} \\
v_{d s}+\frac{2}{3} \mu r_{s} i_{f} \cos \theta & =r_{s} i_{d s}+\frac{d \lambda_{d s}}{d t}+\omega \lambda_{q s} \\
v_{0 s}+\frac{1}{3} \mu r_{s} i_{f} & =r_{s} i_{0 s}+\frac{d \lambda_{0 s}}{d t}
\end{aligned}
$$

$\mu$ é o percentual de espiras em curto; $\lambda_{d s}, \lambda_{q s}$ e $\lambda_{0 s}$ são os enlaces de fluxo do estator das componentes $d q ; i_{d s}, i_{q s}$ e $i_{0 s}$ são as correntes de estator das componentes $d q ; \omega$ a velocidade do eixo $d q ; r_{s}$ a resistência do estator.

O enlace de fluxo $\lambda_{a s_{2}}$ e a tensão $V_{a s}$ nas espiras em curto são calculados conforme 4 e 5 respectivamente.

$$
\begin{array}{r}
\lambda_{a s_{2}}=\mu L_{l s}\left(i_{q s} \operatorname{sen} \theta+i_{d s} \cos \theta-i_{f}\right)+ \\
\mu L_{m}\left(i_{q s} \operatorname{sen} \theta+i_{d s} \cos \theta+i_{q r} \operatorname{sen} \theta+i_{d r} \cos \theta-\frac{2}{3} \mu i_{f}\right) \\
V_{a s_{2}}=\mu r_{s}\left(i_{d s} \cos \theta+i_{q s} \operatorname{sen} \theta-i_{f}\right)+\frac{d \lambda_{a s^{2}}}{d t}=r_{f} i_{f}
\end{array}
$$

$L_{l s}$ e $L_{m}$ são respectivamente a indutância de dispersão do estator e indutância mútua. 
O conjugado do motor pode ser calculado por 6 .

$$
\begin{gathered}
T=\frac{3}{2} \frac{P}{2} L_{m}\left(i_{q s} i_{d r}-i_{d s} i_{q r}\right)+ \\
\frac{P}{2} \mu L_{m} i_{f}\left(i_{q r} \sin \theta-i_{d r} \cos \theta\right)
\end{gathered}
$$

Sendo $P$ o número de pares de polos.

\section{METODOLOGIA}

O fluxograma apresentado na Figura 2 mostra as etapas da metodologia proposta.

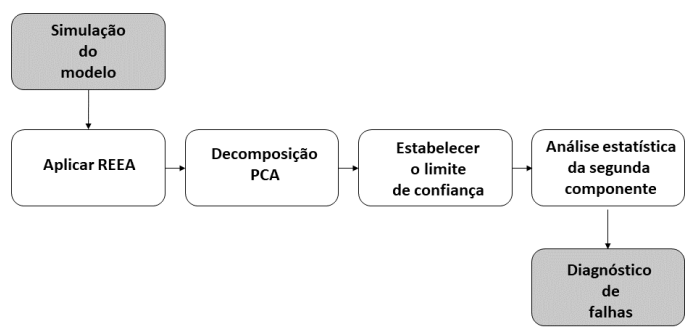

Figura 2. Fluxograma da metodologia proposta.

- Etapa 1: Implementação do modelo de curto-circuito entre espiras proposto por Baccarini et al. (2010), e coleta de dados experimentais.

Nesta etapa deve-se separar os dados sem defeito em dois grupos, um para de definir o limite de confiança, e outro para validação;

- Etapa 2: Extração do resíduo através do processo de suavização da corrente de partida, usando REEA;

- Etapa 3: Aplicar a PCA em cada um dos sinais de corrente suavizados e obter a segunda componente de cada um dos sinais;

- Etapa 4: Estabelecer um limite de confiança, a partir de dados sem falha.

- Etapa 5: Analisar os sinais de validação por meio das medidas estatísticas (variância e energia estatística);

- Etapa 6: Diagnosticar presença de falha a partir dos resultados obtidos na etapa 5, se estiver acima do limiar o motor está com falha, se estiver abaixo do limar não ocorreu falha.

\subsection{Média dos Envelopes Extremos Robusta}

A média dos envelopes extremos robusta (Robust Extreme Envelope Average - REEA) desenvolvida por Batista and Silveira (2012) baseia-se em médias sucessivas entre os envelopes superior e inferior, durante o processo de médias parte do ruído contido no sinal é eliminado.

Porém, neste trabalho a técnica será utilizada para separar do sinal de corrente as altas oscilações, que carregam as informações de falha, das baixas oscilações e não para suavização do sinal.

A REEA segue os seguintes passos:

Passo 1: Identificar os máximo e mínimos locais do sinal de corrente de partida;

Passo 2: Encontrar os envelopes superior e inferior, por um processo de interpolação dos pontos de máximos e mínimos locais;

Passo 3: Calcular a média entre os dois envelopes. Este novo sinal obtido representa o sinal suavizado;

O sinal utilizado para o diagnóstico, sinal que contém as altas oscilações, é obtido realizando a diferença entre o sinal de corrente original e o sinal suavizado obtido em 3 .

O critério de parada que determina número de iterações realizadas, ou seja, quantas vezes a média é calculada, deve ser definido a priori. Neste caso foi escolhido a Relação sinal-ruído (SNR), enquanto o resíduo for maior que $5 \mathrm{db}$ o processo de suavização continua.

\subsection{Analise de Componente Principal}

A PCA é uma técnica estatística que transforma linearmente um conjunto de variáveis originais em um outro consideravelmente menor, de variáveis não correlacionadas que representa a maioria das informações do conjunto original de variáveis Jolliffe (1986). A PCA será utilizada como uma abordagem para isolar características das componentes de falha e não como no seu contexto convencional para a redução de dimensionalidade.

A PCA realiza uma transformação do espaço original de tal forma que, sob a nova representação, os dados não estão mutuamente correlacionados. Os eixos do novo sistema de coordenadas apontam para as direções de maior variação dos dados. As novas variáveis, denominados componentes principais são ordenadas por variância: A primeira componente, representa a direção da maior variação dos dados, a direção do segundo componente, representa o maior da variância restante ortogonal ao primeiro componente Scholz (2006).

A PCA utilizada contém duas componentes principais, mas pode ser naturalmente estendida para obter o número necessário de componentes que, em conjunto, abrangem o espaço cobrindo a quantidade desejada de variância. Como as componentes descrevem direções específicas no espaço de dados, cada componente é uma combinação linear de todas as variáveis originais.

A PCA consiste em uma série de etapas:

Passo 1: Carregar os vetores de dados;

Passo 2: Subtrair a média dos sinais do conjunto de dados; Passo 3: Encontrar a matriz de covariância do conjunto de dados;

Passo 4: Encontrar o(s) autovetor(es) e autovalor(es) da matriz de covariância;

Passo 5: Projetar o conjunto das 2 componentes principais que são ordenadas por variância.

A primeira componente que contém informações corrente de partida do motor, e a segunda componente, formada pelo subespaço residual carrega informações dos ruídos, que não são relacionadas ao sistema Chiang et al. (2000). No caso de ocorrência de falha no motor as características irão se manifestar neste espaço residual, portanto o componente utilizada para analise no disgnóstico de falha é a segunda componente principal, que representa a variância restante ortogonal à primeiro componente. 
A analise da componente residual é uma etapa relevante no contexto deste trabalho, podendo ser realizada utilizando métodos estatísticos como por exemplo $T^{2}$-Hotelling e Q-statistic Harrou et al. (2016), redes neurais artificiais Palácios et al. (2016), LDA Park et al. (2007), entre outros. Neste estudo a segunda componente é monitorada através da variância e de uma analogia com a energia estatística implementando o teste de Szekely \& Rizzo's Székely and Rizzo (2013).

Para o diagnóstico da falha é preciso analisar a distância entre os pontos de uma nova amostra com o seu provável valor estatístico. Estabelecer limite de confiança, é uma maneira analítica de encontrar amostras que não seguem o padrão característico determinado, ou seja, valores que excedem o limite estabelecido, indica a ocorrência de falha, caso contrário, não há falha.

Para o cálculo deste limite deve ser separado um número "n" de sinais sem falhas. Aplicar REEA para extrair de cada sinal de corrente as altas oscilações e em seguida aplica-se a transformada PCA nos novos sinais temporais obtidos. Calcula-se a variância de cada uma das "n" segundas componentes obtidas. Então, calcula-se o valor médio e o desvio padrão das variâncias obtidas. O limite é definido como a média somado com uma vez o valor do desvio padrão. Para definir o limiar a limite a ser usado na análise com energia estátistica basta repetir o processo.

A Equação 7 representa o calculo realizado para definir o limite, onde ME é a Medida Estatística, variância ou energia estatística.

$$
\begin{array}{r}
\text { limite }=\text { média }[M E(1)+M E(2)+ \\
\ldots+M E(n)]+1 \cdot \operatorname{desvio}[M E(1)+ \\
M E(2)+\ldots+M E(n)]
\end{array}
$$

\section{SIMULAÇÕES E DISCUSSÕES}

Para a validação computacional da metodologia proposta foi utilizado uma máquina trifásica de 4 polos, potência $3 \mathrm{HP}$, Tensão 220V, Velocidade $1724 \mathrm{rpm}$ e Corrente nominal 5,8A obtido em Krause et al. (2013).

Optou-se pelos seguintes condições de simulação:

- Condições de carga: 30\%, 75\% e 100\%;

- Percentual de espiras em curto-circuito: 2\%,4\%,6\% e $8 \%$;

Para cada condição de carga foram realizados 32 sinais de cada percentual de falha, 22 sinais sem falha para calcular o limite e outras 10 amostras sem falha para validação.

A Figura 3 mostra a segunda componente principal obtida da simulação. Observa-se que a característica de falha fica mais nítida a medida que o percentual de espiras com falha aumenta.

Para ilustrar, as Figuras 4 e 5 mostram o teste de classificação de 32 sinais com $4 \%$ de falha, 32 sinais com $8 \%$ de falha e 10 sem falha da corrente de estator, operando em condição nominal de carga, usando respectivamente, variância e energia estatística para classificação.

A Tabela 1 mostram a classificação das 32 sinais, para todos os níveis de falha e condições de carga definidas.

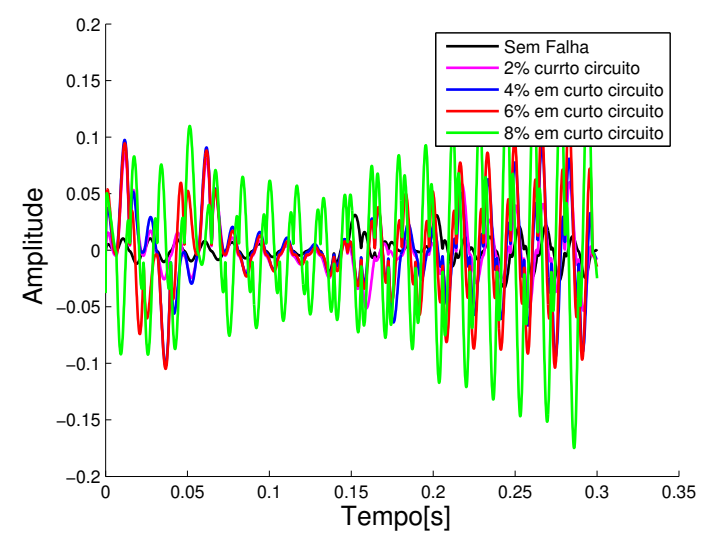

Figura 3. Segunda componente da corrente sem falha, $2 \%$, $4 \%, 6 \%$ e $8 \%$ de espiras em curto.

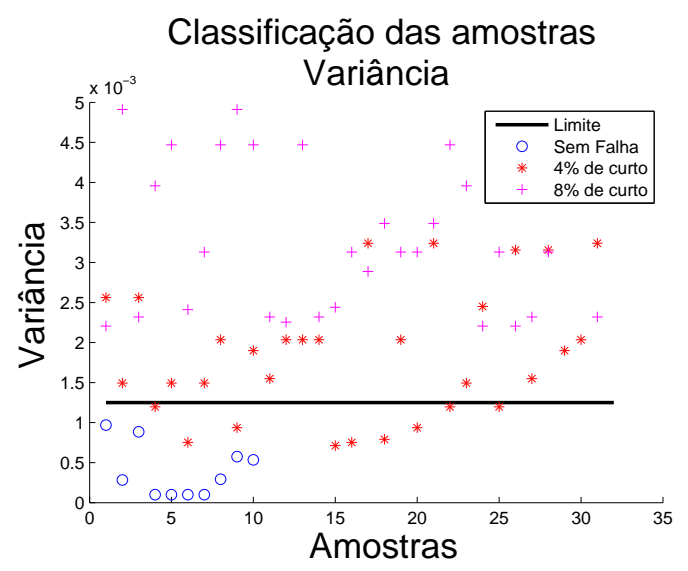

Figura 4. Classificação dos sinais sem falha, $4 \%$ e $8 \%$ de curto, usando o variância para classificação.

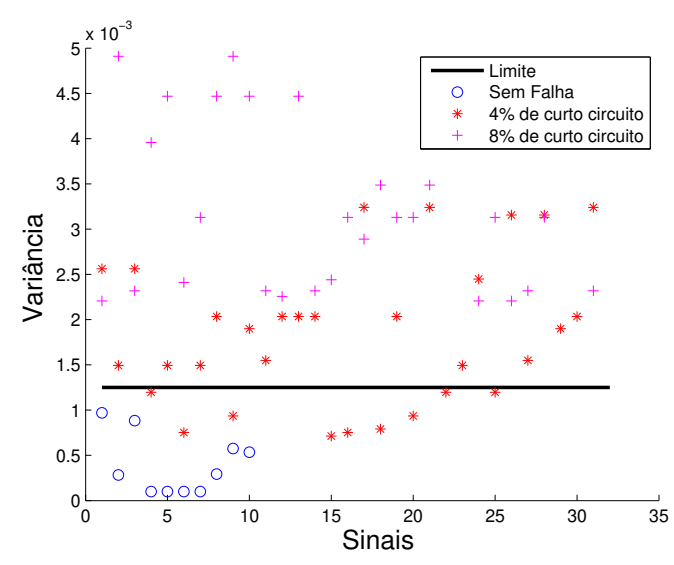

Figura 5. Classificação dos sinais sem falha, $4 \%$ e $8 \%$ de curto, usando o energia estatística para classificação.

Observa-se que, quanto maior a carga acoplada ao eixo do motor maior é a capacidade do método em diagnosticas corretamente falhas iniciais de curto-circuito entre espiras. Também nota-se que em níveis de carga analisados que conforme o percentual de falha aumenta melhor é a capacidade do método em diagnosticar a falha. 
Tabela 1. Classificação dos sinais sem falha e com os diferentes níveis de falha em todas as condições de operação do motor definidas para a simulação computacional.

\begin{tabular}{|c|c|c|c|c|}
\cline { 3 - 5 } \multicolumn{2}{c|}{} & \multicolumn{3}{c|}{ Carga } \\
\hline \multirow{2}{*}{$\begin{array}{c}\text { curto } \\
\text { circuito }\end{array}$} & $\begin{array}{c}\text { Classifi- } \\
\text { cação }\end{array}$ & $30 \%$ & $75 \%$ & $100 \%$ \\
\hline \multirow{2}{*}{$3 \%$} & Variância & 41,02 & 41,02 & 66,66 \\
\cline { 2 - 5 } & Energia & 43,58 & 53,84 & 66,66 \\
\hline \multirow{2}{*}{$8 \%$} & Variância & 53,84 & 56,41 & 76,92 \\
\cline { 2 - 5 } & Energia & 74,35 & 56,41 & 76,92 \\
\hline \multirow{2}{*}{$13 \%$} & Variância & 66,66 & 74,35 & 100 \\
\cline { 2 - 5 } & Energia & 94,87 & 100 & 100 \\
\hline \multirow{2}{*}{$18 \%$} & Variância & 94,87 & 100 & 100 \\
\cline { 2 - 5 } & Energia & 94,87 & 100 & 100 \\
\hline
\end{tabular}

\section{RESULTADOS EXPERIMENTAIS}

Para avaliar experimentalmente o desempenho da metodologia proposta, testes experimentais foram realizados em laboratório utilizando um motor de indução trifásico, 3 $\mathrm{HP}, 220 \mathrm{~V}, 60 \mathrm{~Hz}, 8,7 \mathrm{~A}$, com 4 polos e velocidade nominal de $1730 \mathrm{rpm}$.

O motor foi acoplado diretamente a uma máquina de corrente contínua que atua como uma carga. O controle de diferentes níveis de carga pode ser realizados alterando a corrente de excitação do gerador CC, a alimentação do campo é feita através de um varivolt monofásico ligado a um retificador. Para configurar um baixo percentual de carga acoplado no eixo do motor, aproximadamente $30 \%$ do torque nominal, a tensão de alimentação do gerador foi fixada em 70V. Figura 6 mostra a bancada de testes usada.

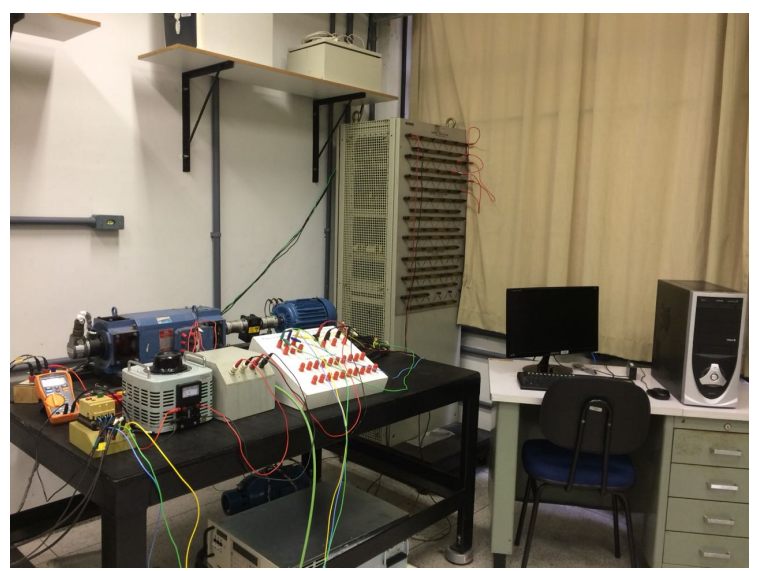

Figura 6. Bancada experimental.

Para a aquisição dos sinais de corrente, foi utilizada uma sonda de corrente (A622 AC/DC - $100 \mathrm{~Hz}$ Tectronix), placa de aquisição (NI PCI- 4461 da National Instruments, e o software VibSoft-80, configurado para uma frequência de $10,24 \mathrm{kHz}$ e tempo de gravação de 0,2 segundos. O filtro anti-aliasing foi configurado para o tipo passa-baixa com frequência de corte de $2,5 \mathrm{kHz}$.

Foram coletadas 32 sinais de corrente sem falha, divididas da seguinte forma:22 para o calculo do limite e 10 para validação. Outras 32 sinais foram coletadas com $5 \%$ das espiras em curto-circuito.

A Figura 7 mostra a segunda componente dos dados experimentais. Nota-se um aumento nas amplitudes das componentes que carregam a característica de falha em relação as que não tem falha.

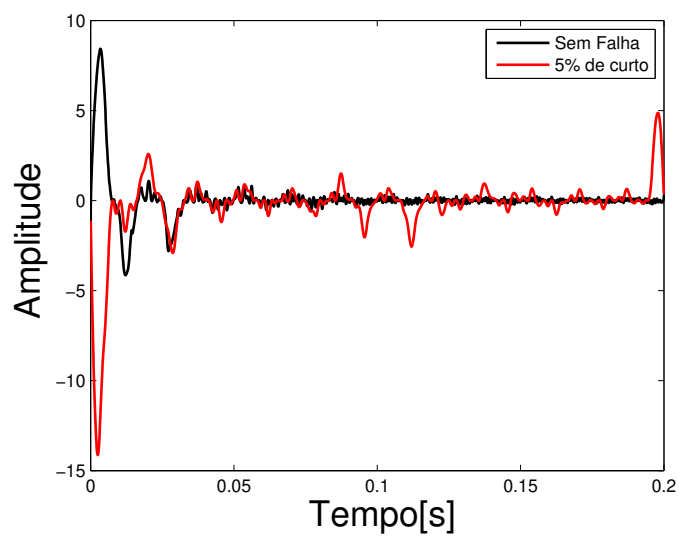

Figura 7. Segunda componente da corrente sem falha, e com $5 \%$ das espiras em curto-circuito.

As Figuras 8 e 9 mostram o teste de classificação dos 42 sinais coletadas (32 com falha e 10 sem falha), variância e energia estatística para classificação respectivamente.

Apenas dois sinais se encontraram fora do modelo PCA indicando alarmes falso positivo, e algumas amostras que deveriam caracterizar falhar indicaram falsos negativos.

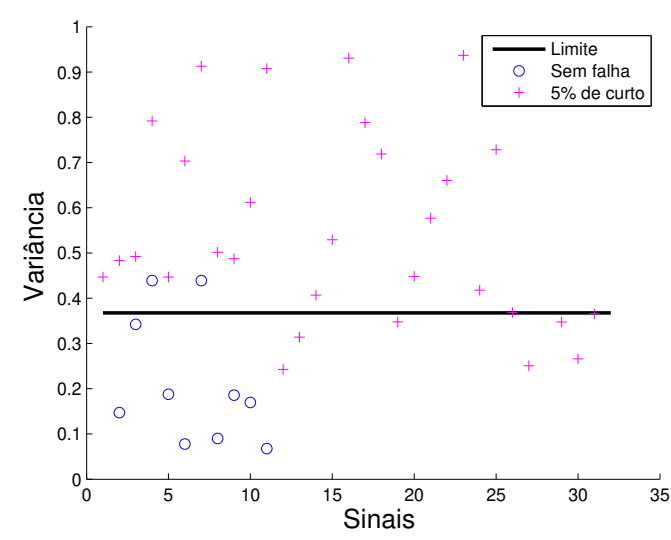

Figura 8. Classificação dos sinais experimentais sem falha e com falha. Utilizando Variância para classificação.

A Tabela 2 mostra a classificação de todos os sinais coletados aplicando a PCA direto na corrente transitória, e a PCA aplicada no resíduo extraído através do método REEA.

Tabela 2. Classificação dos sinais sem falha e com falha em todas as condições de operação do motor definidas para os testes experimentais.

\begin{tabular}{|c|c|c|c|}
\hline $\begin{array}{c}\text { Curto- } \\
\text { circuito }\end{array}$ & $\begin{array}{c}\text { Classi- } \\
\text { ficação }\end{array}$ & $\begin{array}{c}\text { Sem } \\
\text { extração } \\
\text { de resíduo }\end{array}$ & $\begin{array}{c}\text { Com } \\
\text { extração } \\
\text { de resíduo }\end{array}$ \\
\hline \multirow{2}{*}{$5 \%$ de curto } & Variância & 43,90 & 80,48 \\
\cline { 2 - 4 } & Energia & 46,34 & 92,68 \\
\hline
\end{tabular}

A extração do resíduo da corrente transitória do motor foi essencial para os resultados obtidos, aumentando em 


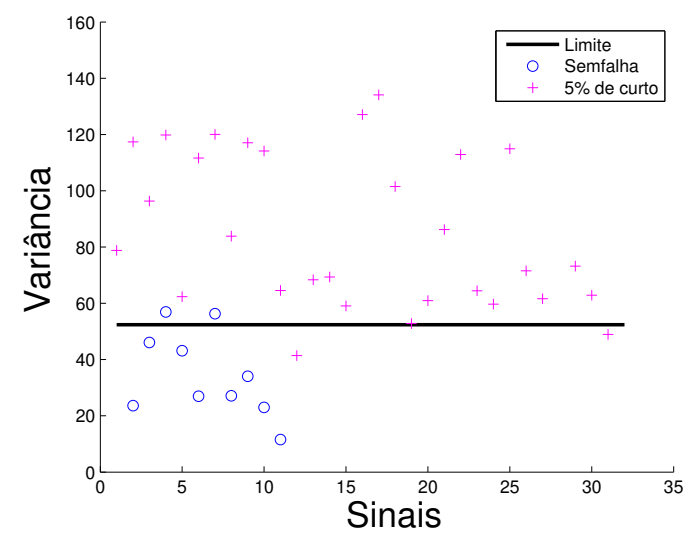

Figura 9. Classificação dos sinais experimentais sem falha e com falha. Utilizando energia estatística para classificação.

mais de $30 \%$ o percentual de acerto dos dados testes experimentais.

\section{CONCLUSÕES}

A técnica desenvolvida mostrando mostrou-se adequada para diagnóstico de curto-circuito entre espiras. Apresentando como vantagem a identificação de falhas de baixa intensidade utilizando a corrente de partida do motor, tendo a possibilidade de aplicação da técnica quando o motor operar com cargas variáveis e/ou tiver um curto tempo de operação por não depender de um grande tempo de aquisição. O percentual de diagnósticos corretos do método aumenta conforme evolução da falha.

O método de classificação utilizando o cálculo da Energia Estatística apresenta uma relevância no percentual de acerto em ralação ao método baseado na variância.

O método proposto utilizando a PCA apresentou baixo custo computacional, é uma ferramenta versátil para a aplicação prática em uma planta industrial, uma vez que requer apenas a medição da corrente de partida em uma fase de alimentação.

\section{AGRADECIMENTOS}

Os autores agradecem à UFSJ, à FAPEMIG e a CAPES pelo apoio financeiro para a realização do trabalho.

\section{REFERÊNCIAS}

Araújo, R., Rodrigues, R., de Paula, H., and Baccarini, L. (2010). Desgaste prematuro e falhas recorrentes dos rolamentos de um mit: Estudo de caso. In Industry Applications (INDUSCON), 2010 9th IEEE/IAS International Conference on, 1-6. IEEE.

Baccarini, L.M.R., de Menezes, B.R., and Caminhas, W.M. (2010). Fault induction dynamic model, suitable for computer simulation: Simulation results and experimental validation. Mechanical Systems and Signal Processing, 24(1), 300-311.

Batista, F.B. and Silveira, M.E. (2012). Noise reduction on beam modes using extreme envelope average. Technical report, SAE Technical Paper.
Bonnett, A.H. and Yung, C. (2008). Increased efficiency versus increased reliability. IEEE Industry Applications Magazine, 14(1).

Boqiang, X., Heming, L., and Liling, S. (2003). Apparent impedance angle based detection of stator winding interturn short circuit fault in induction motors. In Industry Applications Conference, 2003. 38th IAS Annual Meeting. Conference Record of the, volume 2, 1118-1125. IEEE.

Chiang, L.H., Russell, E.L., and Braatz, R.D. (2000). Fault detection and diagnosis in industrial systems. Springer Science \& Business Media.

Cusido, J., Romeral, L., Ortega, J.A., Rosero, J.A., and Espinosa, A.G. (2008). Fault detection in induction machines using power spectral density in wavelet decomposition. IEEE Transactions on Industrial Electronics, 55(2), 633-643.

Douglas, H., Pillay, P., and Ziarani, A. (2005). Broken rotor bar detection in induction machines with transient operating speeds. IEEE transactions on energy conversion, 20(1), 135-141.

Ferreira, F.J. and de Almeida, A.T. (2016). Energy savings potential associated with stator winding connection mode change in induction motors. In Electrical Machines (ICEM), 2016 XXII International Conference on, 2775-2783. IEEE.

Georgoulas, G., Mustafa, M.O., Tsoumas, I.P., AntoninoDaviu, J.A., Climente-Alarcon, V., Stylios, C.D., and Nikolakopoulos, G. (2013). Principal component analysis of the start-up transient and hidden markov modeling for broken rotor bar fault diagnosis in asynchronous machines. Expert Systems with Applications, 40(17), 7024-7033.

Harrou, F., Ramahaleomiarantsoa, J.F., Nounou, M.N., and Nounou, H.N. (2016). A data-based technique for monitoring of wound rotor induction machines: A simulation study. Engineering Science and Technology, an International Journal, 19(3), 1424-1435.

Jolliffe, I.T. (1986). Principal component analysis and factor analysis. In Principal component analysis, 115128. Springer.

Krause, P., Wasynczuk, O., Sudhoff, S.D., and Pekarek, S. (2013). Analysis of electric machinery and drive systems, volume 75. John Wiley \& Sons.

Palácios, R.H.C., Goedtel, A., Godoy, W.F., and Fabri, J.A. (2016). Fault identification in the stator winding of induction motors using pca with artificial neural networks. Journal of Control, Automation and Electrical Systems, 27(4), 406-418.

Park, W.J., Lee, S.H., Joo, W.K., and Song, J.I. (2007). A mixed algorithm of pca and lda for fault diagnosis of induction motor. In International Conference on Intelligent Computing, 934-942. Springer.

Scholz, M. (2006). Approaches to analyse and interpret biological profile data. Universitat Potsdam.

Székely, G.J. and Rizzo, M.L. (2013). Energy statistics: A class of statistics based on distances. Journal of statistical planning and inference, 143(8), 1249-1272.

Watson, J. and Paterson, N. (1998). Improved techniques for rotor fault detection in three-phase induction motors. In Industry Applications Conference, 1998. Thirty-Third IAS Annual Meeting. The 1998 IEEE, volume 1, 271277. IEEE. 